\title{
Atomic Samples in Resonators: Forces, Photons, Feedback
}

\author{
James K. Thompson, Adam T. Black, and Vladan Vuletić \\ Department of Physics, MIT-Harvard Center for Ultracold Atoms, and Research \\ Laboratory of Electronics, MIT, Cambridge, Massachusetts 02139, USA
}

\begin{abstract}
We analyze laser cooling of polarizable particles (classical light scatterers) using continuous dispersive position detection and active feedback with an optical resonator. We derive a simple expression for the cooling force, and show that its magnitude is proportional to the Rayleigh scattering rate into the detector. The velocity dependence of the force is determined exclusively by the frequency dependence of the loop gain. In particular, it is possible to design simple feedback loops that significantly outperform the Doppler force in terms of the combination of velocity capture range and final temperature.
\end{abstract}

In proton-antiproton accelerators, a significant increase in beam brightness and collision rates has been attained with stochastic cooling. This method, proposed by van der Meer [1], is essentially a feedback scheme where corrective forces are applied in response to observed thermal fluctuations of the particle beam. Although the applied forces are electrostatic, and as such conservative, the conditional application of such forces in response to an observed signal renders them dissipative. Stochastic cooling does not violate the second law of thermodynamics, since the observation increases the entropy of the total world by more than the cooling of the beam reduces it, as shown by Szillard [2]. Nevertheless, one may wonder how the cooling is reconciled with Liouville's theorem that forbids compression in phase space by conservative forces. One answer is that a finite-number system is represented by a point in phase space, and that it is possible to observe the location of this point, and move it towards a desired region in phase space [1].

The name 'stochastic cooling' is associated with a many-particle viewpoint in which stochastic fluctuations of the sample are measured, and subsequently reduced by an applied force. However, the technique is not any more 'random' than other cooling methods. In stochastic cooling, a measured signal deterministically propagates through a (linear) feedback loop to generate a correction force, and the total signal from the sample is simply a superposition of the signals generated by the individual particles. Viewed from this single-particle viewpoint, each particle generates its own feedback signal. However, the random motion of the other particles generates noise at the signal frequencies of the test particle, thereby limiting the maximum loop gain and cooling speed [1].

Since its conception, stochastic cooling has been proposed and analyzed for different objects, both in the classical and quantum domains $[3,4,5,6,7,8,9,10]$. It has been demonstrated experimentally in several systems, including a resonant gravity gradiometer [11], a vibration mode of a mirror [12, 8], and the axial oscillation of a single electron in a Penning trap [13]. For atoms, Mark Raizen and coworkers have proposed to measure fluctuations in the center-of-mass velocity of a sample by means of 
Raman transitions between different velocity classes, and to apply optical dipole forces with far detuned, focused laser beams to reduce the center-of-mass momentum [14]. Their numerical simulations indicate that such a scheme should produce observable cooling, and that the necessary cooling time could be shortened with finer spatial resolution of the measurement and the correction force.

Here we analyze cooling by active feedback of a sample of polarizable particles inside a resonator. The basic idea is the same as in Raizen's optical stochastic cooling method [14], and in a recent quantum mechanical simulation of single-atom cooling inside an optical resonator in the strong coupling limit [10]. However, we calculate the cooling force in the classical limit of coherent scattering, i.e., low saturation of atomic transitions, and derive simple analytical formulas that can be easily interpreted in terms of the frequency-dependent loop gain. Our analysis shows that, similar to cooling by coherent scattering inside a resonator without active feedback (cavity Doppler cooling $[15,16,17])$, the cooling force is proportional to the rate at which the atom coherently scatters photons into free space (Rayleigh scattering rate), but it is otherwise independent of the atomic level structure. In particular, we show that for active-feedback cooling the velocity dependence of the cooling force and its sign are determined exclusively by the frequency-dependent loop gain. Surprisingly, it is possible to design simple feedback loops that significantly outperform Doppler cooling in that they combine a large zero-velocity friction coefficient (i.e., low final temperatures) with a very wide velocity capture range.

We also find that the performance of external-feedback cooling is determined by the single-atom optical density for the chosen size of the detection beam. Therefore, as already noted by Raizen [14], the cooling performance improves with increased spatial resolution. The dependence on the single-atom optical density implies that cooling in free space, as proposed in Ref. [14], will be very difficult to observe, and that it is more promising to use an optical resonator to enhance the signal. In this case the optical density transforms into the cooperativity parameter; i.e., the signal is enhanced by the finesse of the cavity as the light interacts repeatedly with the atom while circulating in the resonator.

We begin by sketching the basic concepts proposed here. The first requirement for active-feedback cooling is to measure the time evolution of the atomic position. This is accomplished by monitoring the transmission of a probe laser through a standingwave cavity (figure 1). The cavity transmission varies with atomic position because the polarizable atom can be associated with a position-dependent index of refraction that changes the round-trip optical path length inside the cavity [15]. When the atom is at an antinode, it tunes the resonator frequency by a maximum amount, whereas the presence of the atom at a node, where the resonator field is zero, has no influence on the resonator frequency. The atom's time-dependent position along the cavity axis can be determined from the modulation of the transmitted intensity as the cavity tunes closer to or further from resonance with the fixed-frequency probe laser. The frequency of the modulation determines the atomic velocity while the phase of the modulation determines the atomic position with respect to the intracavity standing wave.

Having measured the atom's position and velocity, a corrective force must be generated to reduce the velocity. This cooling force is produced by modulating the power of the detection beam in order to create a modulation of the intensity of the intracavity standing wave. The polarizable atom experiences the optical dipole potential of the standing wave, that in the absence of modulation would yield no net force when aver- 


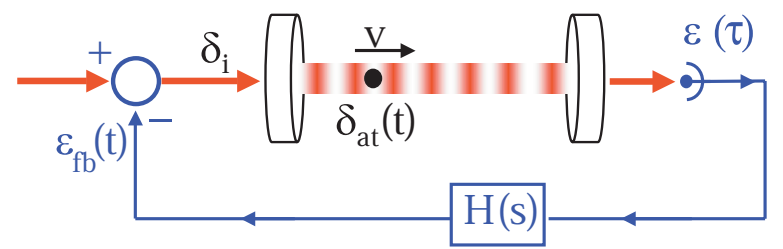

Figure 1: Proposed setup for continuous active-feedback cooling of polarizable particles inside an optical resonator. The moving atom periodically detunes the standingwave resonator by an amount $\delta_{\text {at }}(t)$. The modulation of the cavity transmission, as measured by a fixed-frequency probe beam, is propagated through a linear electronic feedback loop with gain $H(s)$ in the Laplace domain, and applied to the intensity of the probe beam. The modulated probe beam generates a time-varying optical dipole force on the atom, that for appropriate choice of loop gain $H(s)$ cools the atom.

aged over several nodes and antinodes. However, by modulating the potential depth with fixed phase relative to the atomic motion, the atom can be made to move up a larger potential hill than it experiences when moving downhill, leading to a reduction of the atomic velocity $[15,16]$. We will show that the required frequency and phase of the desired modulation are related to the measured cavity transmission signal by a simple phase shift.

In most schemes for feedback cooling, the detection signal and the corrective force are generated by physically separate mechanisms (e.g., separate pick-up coils and deflector plates in accelerators). In contrast, here the cooling force is exerted by the probe laser that also serves to define the error signal. While slightly complicating the analysis, this scheme has the advantage that the measurement and applied force are automatically spatially matched, such that no separate interferometric phase stabilization of the detector and corrective force light is required.

Figure 1 shows the setup that we propose in order to implement active-feedbackinduced cooling of arbitrary polarizable particles. The gaseous sample is placed inside a resonator supporting a $T E M_{00}$ Gaussian mode of waist size $w$. The resonator has finesse $F$ and field decay rate constant $\gamma_{c}$. A laser beam of frequency $\omega=c k$ is incident onto the resonator. For this analysis, we assume that this probe beam is far detuned from any atomic transition. If the electric field inside the cavity on axis has amplitude $2 E_{c}$ at an antinode, an atom with complex polarizability $\alpha$ will experience a dipole potential $U_{0} \cos \left(2 k x_{\text {at }}\right)$ of depth $U_{0}=-\left|E_{c}\right|^{2} R e(\alpha) / 2$. The same atom at position $x_{\text {at }}$ will detune the resonator by an amount $\delta_{\text {at }}=\zeta \gamma_{c} \cos \left(2 k x_{\mathrm{at}}\right)$, where $\zeta=\hbar \eta \Gamma_{\mathrm{sc}} / U_{0}$ is a dimensionless parameter. Here $\eta=6 F /\left(\pi k^{2} w^{2}\right)$ is the singleatom cooperativity parameter, which can be interpreted as the fraction of photons scattered into one direction of the resonant cavity, and $\Gamma_{\mathrm{sc}}=k^{3}\left|\operatorname{Re}(\alpha) E_{c}\right|^{2} /\left(6 \pi \epsilon_{0} \hbar\right)$ is the photon scattering rate into free space, averaged over one period of the intracavity field.

In order to ignore cavity-induced forces $[15,16]$, we assume that the light field inside and the transmission through the resonator adjust quickly to any changes of the incident light power or cavity tuning by the atom. We take the incident light to be detuned relative to the resonator on average by an amount $\delta_{i}=-\gamma_{c}$. The slope of the cavity line is the largest at this point, so that the motion of the atom results in the 
largest transmission modulation signal. If the resonator is detuned by the atom by an amount $\delta_{\text {at }}(t)$ with $\left|\delta_{\text {at }}\right| \ll \gamma_{c}$, the transmitted power changes by a fraction $\varepsilon(t)$ with $|\varepsilon| \ll 1$. The change in transmitted power (measured with a low-noise photodiode) is used as an error signal in a linear feedback loop to adjust the incident intensity by an amount $-\varepsilon_{\mathrm{fb}}(t)$. The fractional change of the intracavity power from its unperturbed value is given by [18]

$$
\varepsilon(t)=-\gamma_{c}^{-1} \delta_{\mathrm{at}}(t)-\varepsilon_{\mathrm{fb}}(t)
$$

In this linear approximation to the cavity transmission, the moving atom modulates the cavity detuning by an amount $\delta_{\text {at }}(t)$, while the feedback loop adjusts the incident intensity by a fraction $-\varepsilon_{\mathrm{fb}}(t)$. If the atom's kinetic energy far exceeds the depth of the optical potential $U_{0}$, the atom's unperturbed motion $x_{\text {at }}=v t$ can be used to calculate the resonator frequency modulation to lowest order, $\delta_{\text {at }}(t)=\zeta \gamma_{c} \cos (2 k v t)$.

To simplify loop stability analysis, we define the feedback loop in terms of the transformed quantities $\tilde{\varepsilon}(s)=L[\varepsilon(t)], \tilde{\varepsilon}_{f b}(s)=L\left[\varepsilon_{\mathrm{fb}}(t)\right]$, where $L$ indicates the Laplace transform. The feedback loop gain $H(s)$ is defined via the relation

$$
\tilde{\varepsilon}_{f b}(s)=H(s) \tilde{\varepsilon}(s) .
$$

In the frequency domain, the complex loop gain at frequency $\omega$ is given by $H(i \omega)$, where the real part $H_{1}(\omega)=\operatorname{Re}(H(i \omega))$ is the in-phase gain, and the imaginary part $H_{2}(\omega)=\operatorname{Im}(H(i \omega))$ is the quadrature gain, i.e., the gain out of phase with the error signal. If the feedback loop is stable, the steady-state solution of Eq. 1 in the time domain exists, and is given by

$$
\varepsilon(t)=-\zeta \frac{\left(1+H_{1}(2 k v)\right) \cos (2 k v t)+H_{2}(2 k v) \sin (2 k v) t}{|1+H(2 i k v)|^{2}} .
$$

The moving atom in the absence of feedback modulates the resonator transmission as $\cos (2 k v t)$. If the feedback loop has non-zero quadrature gain at the Doppler frequency $2 k v$ of the moving atom, it will produce a delayed component $\sin (2 k v t)$ out of phase with the atomic motion. This phase-shifted component introduced by the feedback loop is essential in producing a friction force and cooling. Note that while in conventional laser cooling the delay is due to the atomic response [19], and in cavity cooling due to the resonator $[15,16]$, here the atom and cavity are assumed to react instantaneously, and the delay essential to cooling is produced by the programmable external feedback loop. This delayed component is proportional to the quadrature gain $H_{2}$ at the signal frequency $2 k v$. The characteristic denominator in Eq. (3) arises from the fact that the closed-loop gain, rather than the open-loop gain, determines the system response and cooling force.

Given the modulation of intracavity power $\varepsilon(t)$, it is straightforward to calculate the velocity-dependent force on the atom. In the limit where the atom's kinetic energy far exceeds the potential depth $U_{0}$, work is done on the atom by the modulated periodic potential $V(x, t)=(1+\varepsilon(t)) U_{0} \cos (2 k x)$ at a rate $\dot{W}=\varepsilon(t) 2 k v U_{0} \sin (2 k v t)$ to lowest order. We see that only the quadrature term in Eq. 3 produce a non-zero force when averaged over several nodes and antinodes. The spatially averaged dissipative force $f=\langle\dot{W}\rangle / v$ is given by the simple expression

$$
f(v)=-\hbar k \eta \Gamma_{\mathrm{sc}} \frac{H_{2}(2 k v)}{|1+H(2 i k v)|^{2}} .
$$

The dissipative force is given by the rate at which photons are scattered into the cavity via single-particle Rayleigh scattering $\eta \Gamma_{\mathrm{sc}}$, times the momentum transfered to 

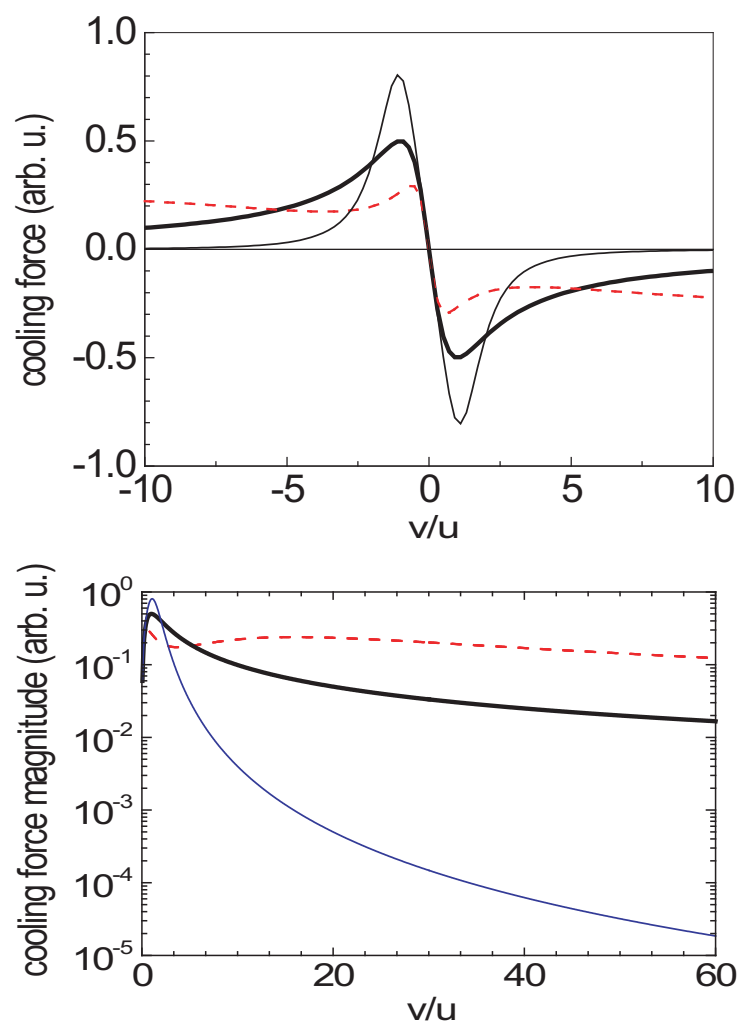

Figure 2: Plots of the cooling force versus velocity to demonstrate the increase in velocity capture range compared to conventional Doppler cooling. Three examples are given: (thin line) conventional Doppler cooling, (thick solid line) feedback with a differentiator loop $H(s)=s$, and (dashed line) feedback with a modified differentiator loop with even larger velocity capture range $H(s)=s(1+s / 10)(1+8 s / 10)$. The unity-gain velocity $u$ has been chosen such that all three forces have the same velocity dependence for small velocities $v$. At large velocities $v \gg u$ the feedback force can exceed the Doppler force by many orders of magnitude. 
the atom per photon $\hbar k$, times a dimensionless function of the atomic velocity that is determined by the frequency dependence of the quadrature gain in closed loop. While the magnitude of the cooling force depends on the strength of the light-atom interaction via the coherent scattering rate $\Gamma_{\mathrm{sc}}$, the sign and velocity dependence of the cooling force are determined exclusively by the frequency-dependent loop gain $H(i \omega)$. Note that since we have assumed in deriving Eq. 3 that a steady-state solution $\varepsilon(t)$ exists, Eq. 4 should only be used to calculate the force for a gain $H(s)$ that results in a stable loop.

It is instructive to analyze the velocity dependence of the friction force $f(v)$ in the case of a loop with zero in-phase gain $H_{1}=0$, as is the case for a differentiator, characterized by loop gain $H(s)=s$ (see Fig. 2). (A positive-feedback integrator $H(s)=-1 / s$ would according to Eq. 4 also produce a friction force, but does not correspond to a stable loop.) For $H_{1}=0$, the cooling force can be written as

$$
f(v)=-\hbar k \eta \Gamma_{\mathrm{sc}} \frac{H_{2}}{1+H_{2}^{2}},
$$

where the gain $H_{2}$ is evaluated at the Doppler frequency for backscattering $2 k v$. We see that the feedback force approaches zero for both very small $H_{2} \ll 1$ and very large $H_{2} \gg 1$ quadrature loop gain. The force is maximized for unity open-loop gain $H_{2}=1$. If we plot the cooling force for the differentiator loop,

$$
f(v)=-\hbar k \eta \Gamma_{\mathrm{sc}} \frac{u v}{u^{2}+v^{2}},
$$

as shown in Fig. 2a, we see that the force has a dispersive line shape, and is maximized at the unity-gain velocity $u$ defined by $H_{2}(2 k u)=1$. For large velocities $v$ the differentiator cooling force approaches zero as $v^{-1}$, much more slowly than the conventional Doppler force that scales as $v^{-3}$ for large $v$ (Fig. 2b).

A simple modification of the differentiator loop results in significantly extended velocity capture range (Fig. 2a, dashed line). If we plot a Bode diagram of the differentiator loop gain (thin straight line in Fig. 3), we recognize that it is possible to increase the force at large velocities $v \gg u$ without changing the low-velocity behavior of the cooling force. To this end, we modify the loop gain in such a way that at high frequencies $\omega \gg 2 k u$ the gain is lower than for the differentiator loop, while maintaining approximately the same phase. This can be achieved by rolling off the loop gain above the unity gain point $\omega_{1}$ to proportional gain at frequency $\omega_{2}$, and back to differentiator gain above some frequency $\omega_{3}$. For atoms at velocities $v \gg \omega_{3} /(2 k)$, this loop appears to be a simple differentiator with some higher unity-gain frequency $\omega_{4}$. Because of the reduced gain at $v \gg u$, the result is a force of greater magnitude than the original differentiator loop with unity-gain frequency $u=\omega_{1} /(2 k)$. As Fig. $2 \mathrm{~b}$ shows, such a feedback loop (dashed line) has significantly larger capture range than a differentiator loop (thick solid line), that already is significantly better than the regular Doppler force (thin solid line). By adding more proportional-differentiator steps, it appears possible to arbitrarily extend the cooling force to larger velocities. For this analysis, a velocity limit is given by the initial assumption that the cavity linewidth be much larger than the atomic Doppler effect $\gamma_{c} \gg 2 k v$. At velocities exceeding $\gamma_{c} /(2 k)$, the analysis must be extended to include delays associated with the cavity linewidth, and the associated cavity cooling forces $[15,16]$.

In Eq. 4, we have written the feedback-induced force in terms of the photon momentum and the photon scattering rate, while, in fact, we have performed a purely 


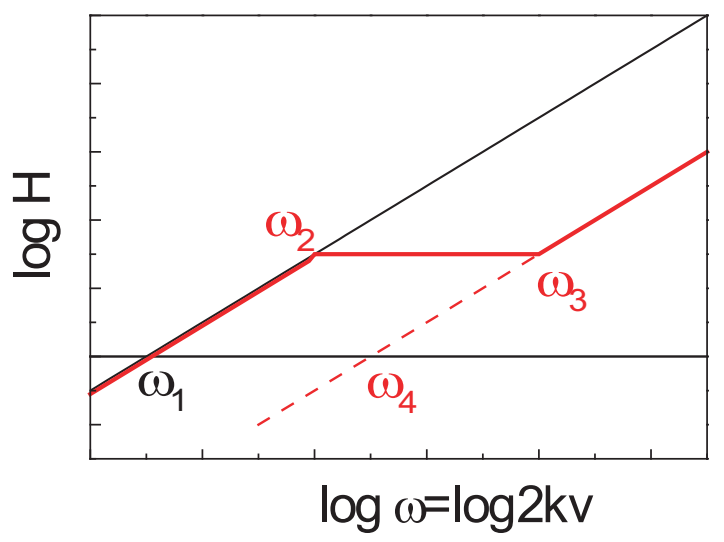

Figure 3: Bode diagram of the open-loop gain illustrating how the velocity capture range can be increased over that of a simple differentiator loop gain $H(s)=s$ (thin straight line). For the differentiator, the cooling force is maximum at the unity-gain point $\omega_{1}$. To extend the cooling force to higher velocities, a modified differentiator loop (thick line) has the gain rolled off to proportional gain at frequency $\omega_{2}$ and rolled back to differentiator gain at frequency $\omega_{3}$. For atoms at Doppler effects much larger than $2 k v \gg \omega_{3}$, the loop is equivalent to a differentiator with larger unity-gain frequency $\omega_{4}$ (dashed line), and consequently larger cooling force at large velocity $v \gg \omega_{3} /(2 k)$. 
classical analysis. This is more obvious if we write the cooling force in the form

$$
f(v)=-\frac{\eta P_{\mathrm{sc}}}{c} \frac{H_{2}(2 k v)}{|1+H(2 i k v)|^{2}}
$$

where $\eta P_{\mathrm{sc}}$ is the Rayleigh-scattered power into the resonator. Classically, any signal, no matter how small, will produce a corresponding cooling force. However, taking the granular nature of the light field into account, the signal will not emerge from the photon shot noise until the classically calculated power emitted into the cavity corresponds to at least one photon energy. The measurement of the cavity transmission can thus be thought of as a homodyne measurement of the forward-scattered field that represents the real signal. Homodyne detection can, however, not be used to improve over the shot-noise limit associated with the direct detection of the forward-scattered photons. This noise limit, arising even if one attempts to cool only one atom, is the main reason we propose to use a resonator for signal enhancement. In free space, very high spatial resolution near the diffraction limit would be necessary to obtain low temperatures with active-feedback cooling.

If one attempts to cool a thermal sample, rather than a single atom, another limit typical of stochastic cooling arises. Namely, for any probe atom moving at velocity $v$ that we attempt to cool at its characteristic frequency $2 k v$, the motion of the other $N$ atoms will produce noise by randomly modulating the cavity frequency. The noise component at $2 k v$ will heat the probe atom. Fortunately, the ensemble-averaged force exerted on the probe particle is zero. Heating of the probe atom results from the fluctuations of the force from zero. The corresponding force fluctuation heating can be calculated using a standard approach [18]. For the differentiator loop we find that the cooling rate is maximized for $u \approx v_{\mathrm{th}}$, and is given by

$$
\gamma_{\mathrm{d}} \approx \frac{k v_{\text {th }}}{6 N}\left(2 \bar{\Gamma}_{\text {cav }}-\bar{\Gamma}_{\text {cav }}^{2}\right)
$$

where the unitless parameter $\bar{\Gamma}_{\text {cav }}=2 N \eta \Gamma_{\mathrm{sc}} \hbar /\left(k_{\mathrm{B}} T\right)$ is just the total scattering rate into the cavity normalized by the sample temperature $T$, and $v_{\text {th }}$ is the thermal velocity. If the collective scattering rate $\bar{\Gamma}_{\text {cav }}$ becomes too large $\left(\bar{\Gamma}_{\text {cav }} \geq 2\right)$, the single-atom picture breaks down since the heating exceeds the cooling. However, it is possible that at this point collective self-organization of the atoms will set in, as observed for cavity-induced forces [20,21], with dramatic consequences for the lightinduced force. Outside this collective regime, the cooling rate is maximized for $\bar{\Gamma}_{\text {cav }}=1$, yielding a rate constant $\gamma_{\mathrm{d}}=k v_{\mathrm{th}} /(6 N)$. This expression shows that the Doppler broadening $2 k v_{\text {th }}$ takes the role of the stochastic-cooling bandwidth, meaning that hotter ensembles can be cooled more quickly. The inverse dependence on the atom number $N$ also shows that the cooling of smaller subsamples can proceed faster, as already noted by Raizen and coworkers [14].

In conclusion, we have derived analytic expressions for the force and cooling rate expected when external feedback is applied to a gaseous sample inside an optical resonator. The cooling force magnitude is proportional to the atom-light interaction, as quantified by the Rayleigh scattering rate into the resonator, while the velocity dependence of the force is determined by the frequency dependence of the quadrature loop gain. In particular, it is possible to design simple feedback loops that significantly outperform the Doppler force in terms of the combination of low-velocity friction coefficient and velocity capture range. The fact that the cooling force relies only on 
Rayleigh scattering implies that particles in different internal states can be cooled simultaneously as long as all states involved have sufficiently large polarizability.

This work was supported in parts by the NSF, the ARO, and the Sloan foundation.

\section{References}

[1] S. van der Meer, Rev. Mod. Phys. 57, 689 (1985), and references therein.

[2] L. Szillard, Z. Phys. 53, 840 (1929).

[3] H. M. Wiseman, Phys. Rev. A 49, 2133 (1994).

[4] H. M. Wiseman and G. J. Milburn, Phys. Rev. A 49, 1350 (1994).

[5] S. Wallentowitz, Phys. Rev. A 66, 032114 (2002).

[6] D. Ivanov, S. Wallentowitz, and I. A. Walmsley, Phys. Rev. A 67, 061401(R) (2003).

[7] A. A. Mikhailichenko and M. S. Zolotorev, Phys. Rev. Lett. 71, 4146 (1993).

[8] S. Mancini, D. Vitali, and P. Tombesi, Phys. Rev. Lett. 80, 688 (1998).

[9] V. I. Balykin and V. S. Letokhov, Phys. Rev. A 64, 063410 (2001).

[10] D. A. Steck, K. Jacobs, H. Mabuchi, T. Bhattacharya, and S. Habib, Phys. Rev. Lett. 92, 223004 (2004).

[11] R. L. Forward, J. Appl. Phys. 50, 1 (1978).

[12] P. F. Cohadon, A. Heidmann, and M. Pinard, Phys. Rev. Lett. 83, 3174 (1999).

[13] B. D’Urso, B. Odom, and G. Gabrielse, Phys. Rev. Lett. 90, 043001 (2003).

[14] M. G. Raizen, J. Koga, B. Sundaram, Y. Kishimoto, H. Takuma, and T. Tajima, Phys. Rev. A 58, 4757 (1998).

[15] P. Horak, G. Hechenblaikner, K. M. Gheri, H. Stecher, and H. Ritsch, Phys. Rev. Lett. 79, 4974 (1997).

[16] V. Vuletić, and S. Chu, Phys. Rev. Lett. 84, 3787 (2000).

[17] V. Vuletić, and A. T. Black, Phys. Rev. A 64, 033405 (2001).

[18] V. Vuletić, A. T. Black, and J. K. Thompson, quant-ph/0410168 (2004).

[19] S. Chu, C. Cohen-Tannoudji, and W. D. Phillips, Rev. Mod. Phys. 70, 685 (1998).

[20] A. T. Black, H. W. Chan, and V. Vuletić, Phys. Rev. Lett. 91, 203001 (2003).

[21] P. Domokos and H. Ritsch, Phys. Rev. Lett. 89, 253003 (2002). 